\title{
Increased inflammation and brain glutamate define a subtype of depression with decreased regional homogeneity, impaired network integrity, and anhedonia
}

\author{
Ebrahim Haroon (10) $1,2,3$, Xiangchuan Chen ${ }^{1,2}$, Zhihao Li ${ }^{2,4,5}$, Thrusharth Patel ${ }^{6}$, Bobbi J. Woolwine ${ }^{1,2}$, Xiaoping P. Hu', \\ Jennifer C. Felger ${ }^{1,2}$ and Andrew H. Miller ${ }^{1,2}$
}

\begin{abstract}
Combined increases in peripheral inflammation and brain glutamate may identify a subtype of depression with distinct neuroimaging signatures. Two contrasting subgroups of depressed subjects - with and without combined elevations in plasma C-reactive protein (CRP) and basal ganglia glutamate (high and low CRP-Glu, respectively) were identified by hierarchical clustering using plasma CRP (indexing peripheral inflammation) and magnetic resonance spectroscopy (MRS)-based measurement of left basal ganglia glutamate. High CRP-Glu group status was associated with greater severity of anhedonia and cognitive and motor slowing. Local- and network-level measures of functional integrity were determined using brain oxygen level-dependent (BOLD)-oscillatory activity and graph theory. Greater decreases in concordance of oscillatory activity between neighboring voxels (Regional Homogeneity 'ReHo', $p<0.01$ ) within the MRS volume-of-interest was associated with the High CRP-Glu subgroup. Using brain-wide, CRP-Glu ReHo contrast maps, a covariance network of 41 regions-of-interest (ROIs) with similar ReHo decreases was identified in the High CRP-Glu group and was located to brain structures previously implicated in depression. The 41-ROI network was further decomposed into four subnetworks. ReHo decreases within Subnetwork4-comprised of reward processing regions — was associated with anhedonia. Subnetwork4 ReHo also predicted decreased network integrity, which mediated the link between local ReHo and anhedonia in the Low but not High CRP-Glu group. These findings suggest that decreased ReHo and related disruptions in network integrity may reflect toxic effects of inflammation-induced increases in extrasynaptic glutamate signaling. Moreover, local BOLD oscillatory activity as reflected in ReHo might be a useful measure of target-engagement in the brain for treatment of inflammation-induced behaviors.
\end{abstract}

\section{Introduction}

Research on major depressive disorder (MDD) has been stymied by within- and between-subject variability. One approach to limiting this variability has been to identify patient subgroups based on pathophysiological processes

Correspondence: Ebrahim Haroon (eharoon@emory.edu)

${ }^{1}$ Emory Behavioral Immunology Program, Winship Cancer Center, Emory University, Atlanta, GA 30322, USA

${ }^{2}$ Department of Psychiatry \& Behavioral Sciences, Emory University School of Medicine, Atlanta, GA 30322, USA

Full list of author information is available at the end of the article. such as increased inflammation ${ }^{1-3}$. Indeed, a distinct subtype of depression characterized by increased inflammation has been proposed ${ }^{4}$. In the brain, inflammation triggers a broad array of neurotransmitter, molecular, neural, and behavioral effects ${ }^{5-10}$. For example, altered glutamate (Glu) neurotransmission in subcortical regions has been a key neurochemical target of inflammation in depression ${ }^{11}$. Previously published data indicate that increased plasma concentrations of the systemic inflammatory marker c-reactive protein (CRP) among subjects with MDD predicted elevated Glu concentrations in left

\section{(c) The Author(s) 2018}

(c) (i) Open Access This article is licensed under a Creative Commons Attribution 4.0 International License, which permits use, sharing, adaptation, distribution and reproduction cc) in any medium or format, as long as you give appropriate credit to the original author(s) and the source, provide a link to the Creative Commons license, and indicate if changes were made. The images or other third party material in this article are included in the article's Creative Commons license, unless indicated otherwise in a credit line to the material. If material is not included in the article's Creative Commons license and your intended use is not permitted by statutory regulation or exceeds the permitted use, you will need to obtain permission directly from the copyright holder. To view a copy of this license, visit http://creativecommons.org/licenses/by/4.0/. 
basal ganglia regions as measured by magnetic resonance spectroscopy (MRS), which in turn predicted greater anhedonia and decreased psychomotor speed, reactiontime, and information processing ${ }^{12}$. Similar results were found among individuals treated with the inflammatory cytokine interferon-alpha ${ }^{12-14}$.

As to the mechanisms by which inflammation influences Glu neurotransmission, inflammatory cytokines increase spillover of Glu from the intra- into the extrasynaptic space by decreasing the ability of astrocytes to clear, buffer and contain Glu, while concurrently impairing mechanisms that remove Glu from the extracellular matrix ${ }^{15,16}$. In addition, glial cells and trafficking macrophages increase surface expression of cystine/glutamate exchanger (Xc)-transporters that extrude Glu into the extrasynaptic space in exchange for cystine ${ }^{11,16,17}$. Of note, these Xc- transporters release large volumes of Glu in dangerously close proximity to extrasynaptic receptorbinding sites ${ }^{18}$. Finally, immune activation increases Glulike molecules such as quinolinic acid, which can promote glial Glu release or impair its reuptake ${ }^{19,20}$. Taken together, inflammation preferentially increases Glu in extrasynaptic compared to intrasynaptic locations ${ }^{15,18,21-23}$. Extrasynaptic Glu freely diffuses into the extracellular space and binds to extrasynaptic $N$-methyl-D-aspartate (NMDA) receptors, leading to chaotic, noisy, incoherent signaling activity in the short-term, and synaptic toxicity by suppressing intracellular survival mechanisms in the long-term ${ }^{18,24-26}$. Nonetheless, neural activity-based biosignatures associated with inflammation-induced Glu dysregulation are not well-characterized.

A primary objective of this work was to identify and contrast two subgroups of depressed subjects with and without combined elevations in plasma CRP and basal ganglia glutamate and examine their impact on neural activity. Investigating neural activity using oscillatory frequency, amplitude or coherence of spontaneous brain oxygen level-dependent (BOLD)-fluctuations during resting-state functional MRI offers a unique opportunity to examine these associations ${ }^{27-29}$. Regional Homogeneity (ReHo)-an activity-based metric of concordance in BOLD-signal fluctuations between neighboring voxels-is an effective estimate of local activity coherence at millimetric resolution ${ }^{29-32}$. Based on the hypothesis that combined increases in inflammation and extrasynaptic Glu can lead to disorderly, incoherent local neural activity, we predicted that concurrent increases in peripheral inflammation and MRS Glu would identify a subgroup of depressed patients with reduced local ReHo in the basal ganglia and potentially other brain regions. Moreover, we hypothesized that decreased ReHo in regions affiliated with reward, salience, and attention would be associated with anhedonia and psychomotor slowing. Finally, using exploratory path analyses, we examined whether altered network-level interactions among the above regions mediate the link between inflammation, Glu, local ReHo, and anhedonia.

\section{Materials and methods Subjects}

Fifty subjects with a diagnosis of MDD confirmed using Structured Clinical Interview for DSM-IV (SCID) and with ages ranging between 21-65 years were screened for inclusion into the study ${ }^{3,34}$. Bipolar depressed subjects were also included due to shared mechanisms of anhedonia, inflammation and Glu dysregulation ${ }^{35}$. Exclusion criteria included unstable medical conditions; cognitive, psychotic or substance abuse disorders; and intake of any psychotropic or immune-altering medications. Detailed information on subject recruitment and evaluation is provided in Supplementary Methods. All participants signed informed consent, and the study was approved $a$ priori by the Institutional Review Board of Emory University. Subjects presented in this manuscript represent a subset of those recruited for NCT01426997.

\section{Behavioral and cognitive assessments}

Depression severity was measured using the Inventory of Depressive Symptoms-Self Reported (IDS-SR) ${ }^{36,37}$. Anhedonia was measured using a 3 -item subscale derived from IDS-SR items \#8, 19, and 21 ("anhedonia") that has been previously validated in other studies ${ }^{12,38,39}$. Psychomotor and cognitive performance were assessed using the Finger Tapping Test ${ }^{40}$; Trails Making Test A (TMT) ${ }^{41}$; Digit Symbol Substitution Test of Wechsler Adult Intelligence Scale ${ }^{42}$; Movement and Reaction times-Simple (SMT, SRT, respectively) and Five-Choice Reaction Time (5CMT, 5CRT, respectively)-and "Stockings of Cambridge" (SOC) Tasks of the Cambridge Neuropsychological Test Automated Battery (CANTAB) ${ }^{43,44}$. Of note, principal components analysis of the SOC yielded performance scores for three dimensions (SOC1, SOC2, SOC3) corresponding to "mean initial thinking time", "mean moves", and "mean subsequent thinking time", respectively (Supplementary Methods).

\section{C-reactive protein}

Blood was sampled between 8 and 10:00 a.m. to limit circadian variation, and plasma was collected and stored at $-80{ }^{\circ} \mathrm{C}$ for batched assay ${ }^{12,39}$. Hs-CRP was measured by the immunoturbidometric method using the Beckman AU 480 chemistry analyzer and Ultra WR CRP reagent kit (Sekisui Diagnostics, LLC, Lexington, MA, USA). Mean inter- and intra-assay coefficients of variation were reliably $<10 \%$. CRP values were log-transformed for normality. 


\section{MRI methods}

\section{MRI methods}

All scans were acquired using Siemens $3 \mathrm{~T}$ Trim-Trio systems (Siemens Medical Systems, Erlangen, Germany).

\section{Acquisition}

Anatomical T1 images were obtained using threedimensional magnetization- prepared rapid gradientecho with settings of repetition time $(T R)=2300$ milliseconds $(\mathrm{ms})$, echo time $(\mathrm{TE})=3.02 \mathrm{~ms}$, time following inversion $(\mathrm{TI})=1100 \mathrm{~ms}$, flip angle $=8^{\circ}$ and voxel size $1 \times 1 \times 1 \mathrm{~mm}^{3}$. Single-voxel MRS data were acquired using a PRESS (point-resolved spectroscopy) technique with $\mathrm{TR}=3000 \mathrm{~ms}, \mathrm{TE}=30 \mathrm{~ms}$, sampling size $=1024,128$ averages. A rectangular voxel sized $20 \times 40 \times 30 \mathrm{~mm}^{3}$ located on the left basal ganglia was used to obtain singlevoxel ${ }^{1} \mathrm{H}-\mathrm{MRS}^{12,13}$. Details of acquisition and voxel location are provided in Supplementary Methods.

\section{Resting-state fMRI images}

Resting-state fMRI images were acquired using a Z-saga EPI-pulse sequence for recovering ventral-frontal signal losses regularly seen in gradient-echo BOLD fMRI at $3.4 \times 3.4 \times 4 \mathrm{~mm}^{3}$ resolution in $30 \times 4$-mm-thick axial slices with the following parameters: $\mathrm{FOV}=220 \mathrm{~mm}, \mathrm{TR}=$ $2950 \mathrm{~ms}, \mathrm{TE} 1 / \mathrm{TE} 2=30 / 67 \mathrm{~ms}, \mathrm{FA}=90^{\circ}$, scan time $=$ $7.4 \mathrm{~min}$ (150 repetitions). Forty-five Subjects were required to look at a fixation cross during scanning.

\section{Post processing MRS data-estimation of absolute Glu concentrations}

MRS metabolites were estimated with water-scaling in LC Model using following settings: spectral bandwidth 0.2-4.0 ppm and 2048 complex points ${ }^{46}$ and corrected for cerebrospinal fluid (CSF) volume fraction using methods published earlier ${ }^{12}$. Methods used for post processing including voxel tissue composition, spectral quality, and absolute quantitation of metabolites are provided in Supplementary Table 1 and Supplementary Methods.

\section{Analysis of resting-state data Preprocessing procedure}

Signal spike, slice-timing shift, and motion were corrected in AFNI (https://afni.nimh.nih.gov) ${ }^{47}$, followed by removal of motion and CSF signals within a general linear model (GLM). Local BOLD oscillatory measures were calculated with the residual time-series data derived from the GLM. Of note, white matter (WM) signal was not removed because WM is a key target of inflammation, Glu dysregulation, and depression ${ }^{48,49}$.

\section{Group comparisons of local BOLD oscillatory data}

Local BOLD oscillatory measures-including ReHo, amplitude of low-frequency fluctuations (ALFF) and resting-state fluctuation amplitude (RSFA)-and their fractionated derivatives were obtained and compared. $\mathrm{ReHo}$ is a metric that estimates the concordance (statistical similarity of spontaneous neural activity) between a given voxel and its spatially adjacent, neighboring voxels within the context of a given time-series ${ }^{29,32}$. ALFF is an amplitude-based index of local oscillations in lowfrequency range $(<0.01-0.1 \mathrm{hz})^{50,51}$ and RSFA utilizes power spectral analysis to estimate vascular reactivity information in spontaneous BOLD activity ${ }^{28}$. Oscillatory data were analyzed in two ways: (1) Volume-of-interest (VOI)-based analysis: Averages were calculated across all voxels in the left BG MRS VOI for individual subjects and compared between the groups and (2) Brain-wide analysis of BOLD oscillatory activity that differed in the VOIbased analysis. For each subject, the ReHo data were converted into standard MNI space and spatially smoothed with FWHM $=4 \mathrm{~mm}$. Group comparisons were performed using AFNI "3dttest++", generating a ReHo-difference map after correcting for multiple-test false-positive errors using Monte Carlo serial simulation followed by $p$-value thresholds specified in recent publications addressing the issue of multiple comparisons ("Clustsim" option, $\left.p_{\text {voxel }} \leq 0.01, p_{\text {cluster }} \leq 0.05\right){ }^{52,53}$.

\section{Network analysis}

For each subject, the residual time-series data were temporally filtered $(0.01-0.1 \mathrm{~Hz})$, converted into MNI space and spatially smoothed (FWHM $=4 \mathrm{~mm}$ ). Average time-series data were calculated for regions-of-interest (ROIs). Cross-correlation analysis among time-series data was preformed using MATLAB (MathWorks, Natick, MA, USA), generating a correlation-coefficient matrix which was further transformed into a Fisher $z$-score matrix. With the $z$-score matrix, graph-theory-based measures of brain connectivity were estimated using the Brain Connectivity Toolbox in MATLAB (https://sites. google.com/site/bctnet $)^{54,55}$. Networks were defined as a collection of nodes (ReHo ROIs), and links (edges) between pairs of ROIs. Weighted, undirected network measures were obtained using adjacency and distance matrices within the brain connectivity toolbox. Eleven measures of network integrity including degree (positive and negative connection strengths); internodal clustering (positive and negative clustering coefficients, transitivity, and modularity); assortativity; distance (path length, global efficiency) and eccentricity (diameter and radius) were measured (details in Supplementary Methods). Using graph theory-based metrics enabled computation of integrity at network and subnetwork-level. In addition, "average connectivity $z$-scores" were also calculated across groups of ROIs analysis by averaging the timeseries. 


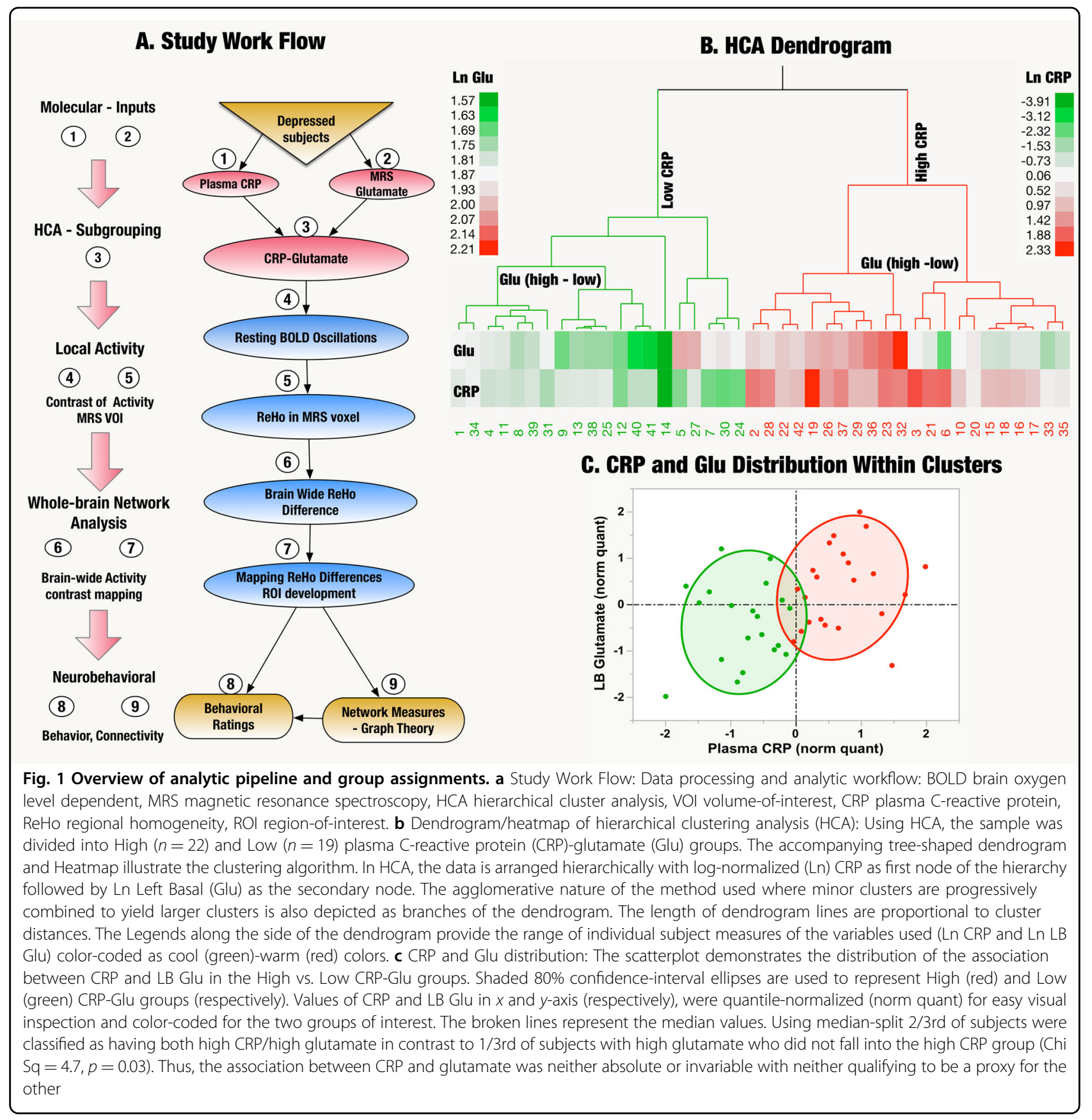

\section{Statistical methods}

Agglomerative Hierarchical Cluster Analysis (HCA) using Ward's linkage method in JMP Pro v13 for Mac (SAS Institute, Cary, NC, USA) was used to classify subjects based on plasma CRP and MRS-based measures of basal ganglia Glu. Both values were log-transformed to scale for uniformity and normality. Cubic Clustering Criterion (CCC-a measure of the cluster numbers providing the best fit) and evaluation of 'screen' plots were used to inform the number of groups. Both CCC $(-1.891$ for 2 cluster solutions vs. $-2.88,-2.61,-2.13$ for 3,4 , and
5 cluster solutions, respectively) and screen plot revealed a two-cluster solution as the most parsimonious model. A dendrogram depicting the cluster differentiation is provided in Fig. 1b. "Variable clustering" in JMP Pro (similar to "varclus" in SAS) was also used to identify groupings of variables based on shared brain signatures ${ }^{56}$. Data were checked for univariate and multivariate normality using standard procedures. Comparisons of background variables were performed using $t$-tests and chi square, and variables that were different between High and Low CRPGlu groups were controlled as covariates in further 
Table 1 Demographic and clinical characteristics of the CRP-Glu groups

\begin{tabular}{|c|c|c|c|}
\hline Group statisticsMean \pm SD & $\begin{array}{l}\text { Low CRP-GLU Cluster } \\
(n=20)\end{array}$ & $\begin{array}{l}\text { High CRP-GLU Cluster } \\
(n=22)\end{array}$ & Test, $p$ \\
\hline \multicolumn{4}{|l|}{ Background and clinical } \\
\hline Age & $38.5 \pm 11.0(122.2)$ & $37.9 \pm 11.3(127.9)$ & $F(1,40)=0.3, p=0.86$ \\
\hline Sex: (Females) \% & $12(40 \%)$ & $18(60 \%)$ & Chi Sq $=2.44, p=0.12$ \\
\hline Race: African Americans, (\%) & $11(44 \%)$ & $14(56 \%)$ & Chi Sq $=0.32, p=0.57$ \\
\hline Education: College grads (\%) & $9(45 \%)$ & $8(36 \%)$ & Chi Sq $=0.32, p=0.57$ \\
\hline Smoker (\%) & $6(30 \%)$ & $4(19 \%)$ & Chi Sq $=0.81, p=0.37$ \\
\hline BMI: Mean (SD) & $26.63 \pm 4.29$ & $35.66 \pm 7.87$ & $\begin{array}{l}F(1,40)=20.7, p< \\
0.001^{\mathrm{a}}\end{array}$ \\
\hline Bipolar/unipolar depression & $1 / 19$ & $1 / 21$ & Chi Sq $=0.005, p=0.94$ \\
\hline Duration of current episode of depression (months) & $170.90 \pm 172.99$ & $204.41 \pm 153.27(23491.87)$ & $F(1,40)=0.44, p=0.51$ \\
\hline Age of onset of depression (Years) & $21.80 \pm 12.67$ & $16.95 \pm 16.95$ & $F(1,40)=1.99, p=0.17$ \\
\hline Number of depressive episodes & $1.89 \pm 3.02$ & $1.38 \pm 0.97$ & $F(1,38)=0.55, p=0.46$ \\
\hline Number of antidepressants used in current episode & $0.95 \pm 2.01$ & $0.73 \pm 1.16$ & $F(1,40)=0.20, p=0.66$ \\
\hline \multicolumn{4}{|l|}{ Behavioral } \\
\hline Anhedonia (IDS3-items) & $4.20 \pm 1.93$ & $5.50 \pm 1.71$ & $F(1,40)=5.3, p=0.03^{\mathrm{a}}$ \\
\hline Response to good/desired events (IDS-SR Item \#8) & $1.25 \pm 0.85$ & $1.82 \pm 0.66$ & $F(1,40)=5.88, p=0.02^{\mathrm{a}}$ \\
\hline Leaden paralysis/physical energy (IDS-SR Item \#30) & $0.95 \pm 0.69$ & $1.64 \pm 0.95$ & $F(1,40)=7.04, p=0.01^{a}$ \\
\hline IDS-SR-Total & $34.35 \pm 7.5$ & $38.2 \pm 8.0$ & $F(1,40)=2.6, p=0.12$ \\
\hline \multicolumn{4}{|l|}{ Cognitive } \\
\hline Trails (total score) & $28.64 \pm 9.73$ & $38.69 \pm 17.38$ & $F(1,40)=5.20, p=0.03^{\mathrm{a}}$ \\
\hline $\begin{array}{l}\text { Sock of Cambridge (initial think time-principal } \\
\text { component) }\end{array}$ & $-0.62 \pm 0.81$ & $0.56 \pm 1.80$ & $F(1,40)=7.19, p=0.01^{a}$ \\
\hline CANTAB 5-choice movement Time (msec) & $468.67 \pm 83.49$ & $554.27 \pm 117.29$ & $F(1,40)=7.29, p=0.01^{a}$ \\
\hline \multicolumn{4}{|l|}{ Neurochemical } \\
\hline Left basal ganglia glutamate & $6.05 \pm 0.68$ & $6.93 \pm 0.96$ & $\begin{array}{l}F(1,40)=11.2, p= \\
0.002^{\mathrm{a}}\end{array}$ \\
\hline \multicolumn{4}{|l|}{ Immune } \\
\hline Plasma CRP (mg/L) & $0.39 \pm 0.23(n=17)$ & $3.99 \pm 2.27(n=20)$ & $\begin{array}{l}F(1,35)=49.6, p< \\
0.001^{\mathrm{a}}\end{array}$ \\
\hline
\end{tabular}

$B M I$ body mass index, IDS-SR Inventory for Depressive Symptoms-Self-Rated Version, anhedonia 3-item Anhedonia Subscale, CANTAB- Cambridge, CRP c-reactive protein (plasma)

${ }^{\text {a }}$ Significant ANOVA

analysis. Canonical, stepwise, discriminant function analysis (DFA) was used to predict membership in High vs. Low CRP-Glu groups using a categorical classifier ( $x$ variable $=$ CRP-Glu status) on known continuous variables (" $y$ " responses). Adaptive elastic regression models in JMP Pro were used to test predictive associations owing to their ability to shrink covariances, limit collinearity, and decrease overall prediction error. Following variable selection (within elastic net models), the predictors and covariates were imported into standard least squares regression models to test for multiple comparisons (using False Discovery Rate corrected (FDR)- $p$-values) and to measure statistical power and estimate effect sizes. Effect sizes were measured using Cohen's $d(95 \% \mathrm{CI})^{57}$, and alternate measures ( $F$-statistic, partial eta, omega, rsquared) were converted into "d" for consistency. Path analysis was performed using Structural Equation Modeling (SEM) protocol in STATA (College Park, Texas, USA). 


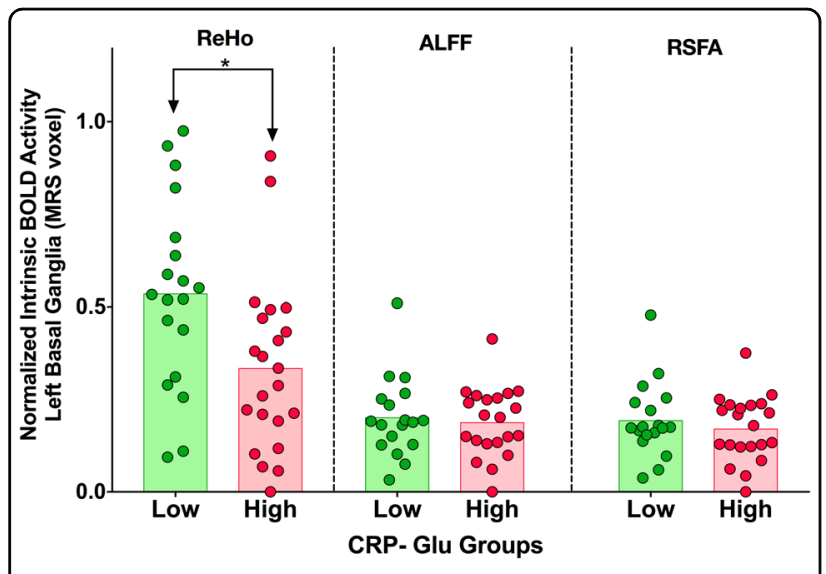

Fig. 2 Local Activity Contrast in the MRS Voxel-of- Interest (VOI). Scatterplot with bar demonstrating differences in plasma CRP and glutamate in the left basal ganglia between High vs. Low CRP-Glu groups. The upper end of the bar is the mean and shaded regions represent range between mean and 0 . Green shade represents Low CRP-Glu and red shades represent High CRP-Glu. Comparison of group means of the three bold oxygen level-dependent (BOLD) indices between (Low-High) CRP-Glu groups revealed that Regional Homogeneity (ReHo) was the only metric of BOLD oscillatory activity that significantly differed between groups: (ReHo). Table 2 provides a detailed summary of individual means and SD

\section{Results}

\section{Sample characteristics}

Of the 50 subjects recruited, eight were excluded [six with poor quality MRS data (metabolite variances $>20 \%$ or motion artifacts, one due to excessive head motion and one for whom resting-state fMRI was not available]. Data from the remaining 42 patients were included in the analyses. Figure 1a illustrates the analytic pipeline used for the study. Using HCA, two clusters-High $(n=22)$ vs. Low $(n=20)$ CRP-Glu groups were identified (Fig. 1b). Both plasma CRP and absolute left basal ganglia Glu were significantly elevated in the High vs. Low CRP-Glu group $(p<0.001$ and 0.002 , respectively). Of note, the crosstabulation of subjects classified into "high" and "low" groups using + above/-below median of CRP and glutamate indicated that $66 \%$ of individuals with high glutamate fell into the high CRP group, while the remaining $33 \%$ of subjects with high glutamate were classified into the low CRP group (Fig. 1c) (Likelihood ratio $=4.76, p=$ 0.03). Thus, the association between CRP and glutamate was neither absolute or invariable with neither qualifying to be a proxy for the other. The samples were wellmatched on all clinical, demographic variables and (right vs. left) handedness other than BMI, which was significantly higher among High vs. Low CRP-Glu subjects $(p<0.001)$. BMI was entered as a covariate in all subsequent analyses (unless otherwise specified). Only $3 /$ 42 subjects were SCID + bipolar depression with 1 and 2 in Low and High CRP-Glu groups, respectively, (Table 1 and Supplementary Table 2).

\section{Behavioral and cognitive variables}

DFA with post hoc analysis of variance (ANOVA) indicated that anhedonia $[F(1,40)=5.34, p=0.03]$, 5CMT $[F(1,40)=7.29, p=0.01]$, TMT $[F(1,40)=5.20, p=0.03]$ and SOC1 $[F(1,40)=7.19, p=0.01]$ were significantly associated with High CRP-Glu status (Table 1). Analysis of individual IDS-SR items indicated that Item\#8, signifying positive valence ["Response of your mood to good or desired events": $F(1,40)=5.53, p=0.02$ ] and item $\# 30$ [Leaden Paralysis/Physical Energy: $F(1,37)=5.80, p=$ 0.02] were also associated with High CRP-Glu status. Most the effect sizes were in the "large" range $(d>0.8)$.

\section{Effect of CRP-Glu status on BOLD-signal metrics in MRS voxel}

DFA with post hoc ANOVA identified reductions in ReHo in the left basal ganglia MRS VOI as the local BOLD oscillatory measure associated with High CRP-Glu status $[F(1,40)=10.20, p=0.003$, canonical $r=0.45]$; with leave-one-out cross-validation indicating a classification-accuracy of 76.2\% (Fig. 2 and Supplementary Table 2). Owing to a lack of similar association, ALFF and RSFA were excluded from further analysis. Testing of 3-way interactions between CRP and glutamate along with covariates (age, sex, race, BMI, smoking) confirmed that neither CRP nor glutamate alone (both $p>0.05$ ) but only (Low-High) CRP-Glu contrast $[\mathrm{PE}=0.83$ (0.34-1.33), Wald Chi $\mathrm{Sq}=10.8, p=0.001]$ predicted ReHo. Of note, the same elastic net model also revealed that race [African American-Caucasian American $=-0.57$ $(-1.08--0.06)$, Wald $=4.82, p=0.03]$ had a significant impact on ReHo decreases and hence was controlled as a covariate in all subsequent analyses.

\section{Whole-brain ReHo and ReHo subnetwork partitioning}

To reliably locate whole-brain ReHo changes within functionally defined space, $10 \mathrm{~mm}$ spheres (\#283) were placed on previously published functional coordinates $^{56,58,59}$, and the spheres demonstrating $>50 \%$ overlap with ReHo-difference map were included as ROIs. A total of 41 ROIs -27 from the first set of 264 ROIs $^{58}$ and an additional set of 17 ROIs curated from depression-related studies $^{56,59}$ that fulfilled the "overlap" criteria were included for further analysis (Fig. 3). Variable clustering approaches were used to decompose the large number of ROIs (\#41) into four subnetworks of ROIs. A detailed description of the ROIs including their coordinates and subnetwork affiliations are provided in Supplementary Table 3. ROI masks were applied to calculate "mean ReHo" of each subnetwork for each individual and grouped for further analyses. 


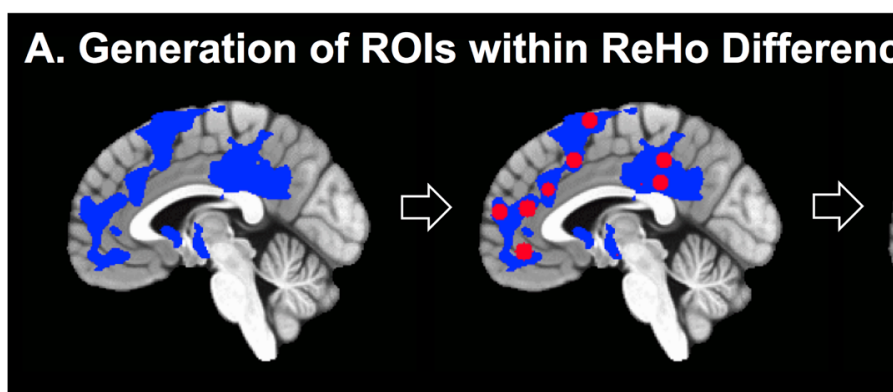

\section{B. Sub-networks of ROls}

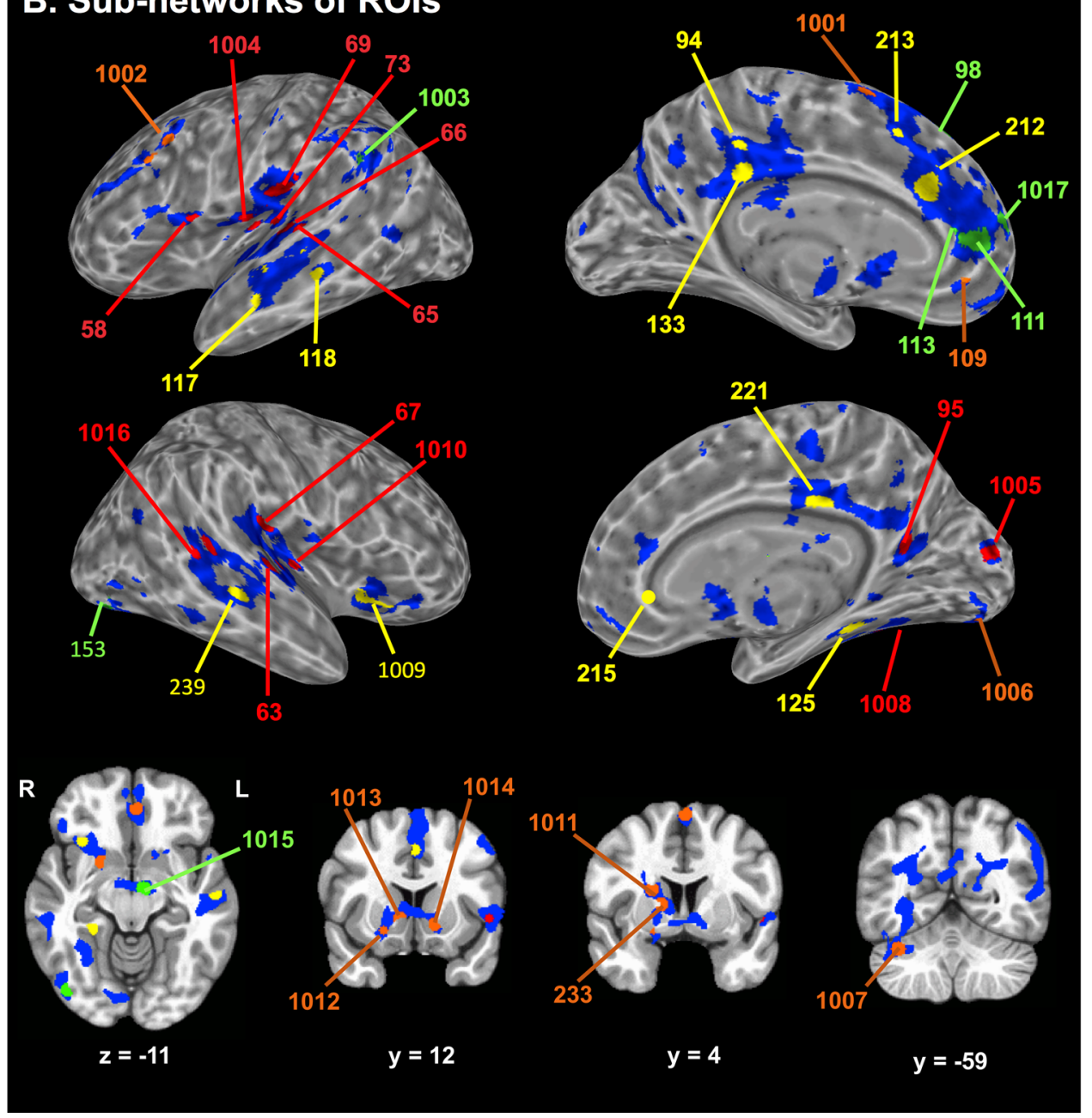

Fig. 3 Regions-of-interest (ROIs) within the ReHo-difference map. a Generation of ROls (right: red spheres) within the ReHo-difference map (left: blue patches): Overlapped portions between the ReHo-difference map and $10 \mathrm{~mm}$ diameter spheres (middle: red circles on blue regions). The background image is a sagittal slice of the MNI-152 brain atlas. b Four sub-networks (SBNs) of ROls shown on the cortical surface (top, middle) and slices (bottom) of the MNI-152 brain. The ROI numbers and their corresponding brain structures are identified in Supplementary Table 3

\section{Subnetwork4 ReHo and behavioral associations}

Subnetwork4 ReHo was the only predictor of anhedonia $[\mathrm{PE}=-20.8, t=-3.76$, FDR $p<0.001, d=1.22(0.55-1.84)]$ and IDS-SR-positive valence Item $\# 8[\mathrm{PE}=-18.1, t=-3.05$, FDR $p=0.004, d=1.03(0.33-1.63]$. Of note, Subnetwork4 included several ROIs within canonical reward and salience networks including ventromedial prefrontal (vMPFC), and dorsal (DS) and ventral striatal (VS) regions (Fig. 3 and Supplementary Table 3$)^{56,59,60}$. None of the other three subnetworks demonstrated significant associations with anhedonia, although Subnetwork1 ReHo was predictor of psychomotor processing speed as measured by TMT [PE $=-0.41, \quad t=-2.71, \quad p=0.01, \quad d=0.85(0.19-1.46)$ and Subnetwork2 ReHo was predictor of IDS-SR leaden 


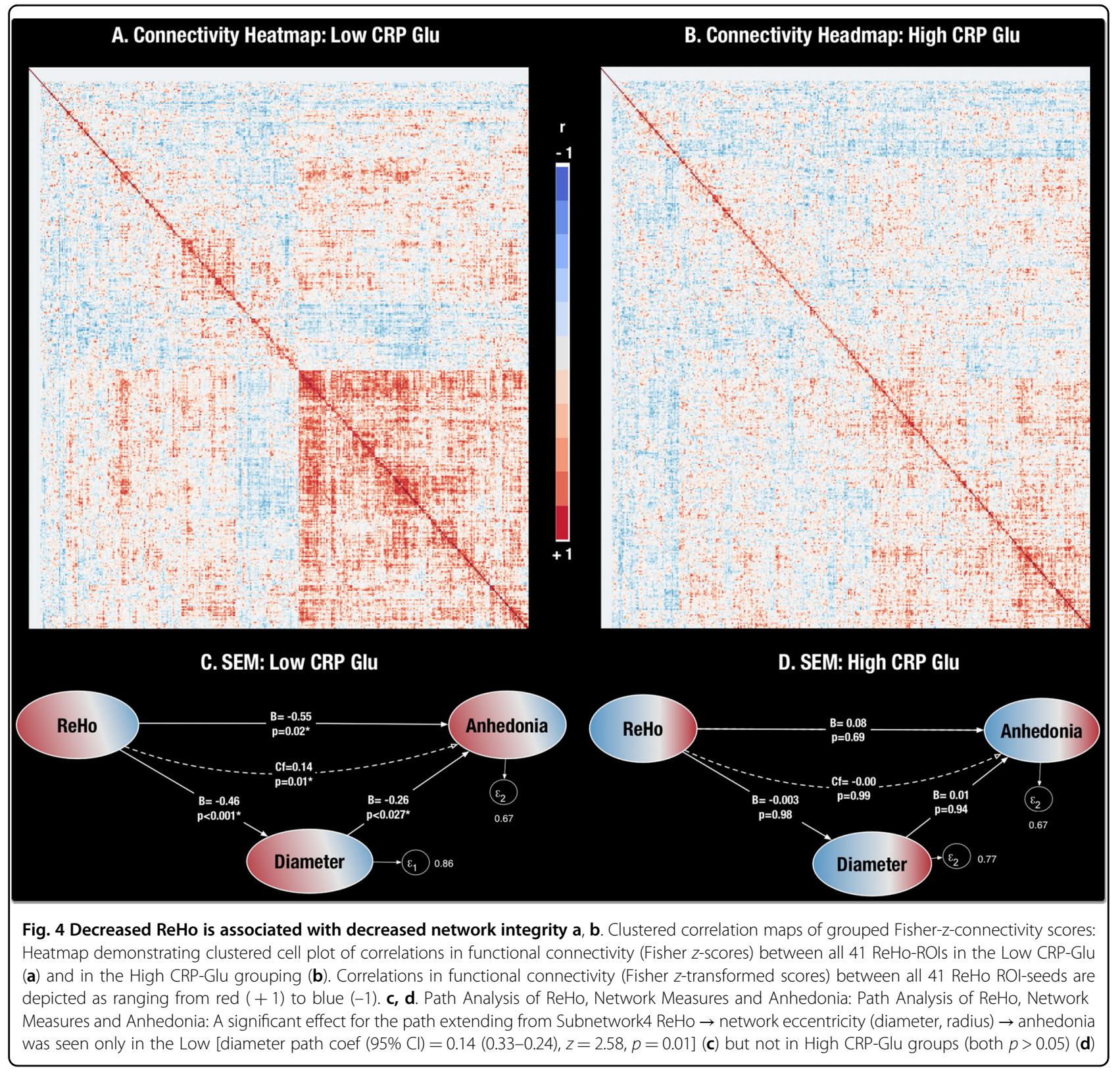

paralysis Item\#30 $[\mathrm{PE}=-0.35, t=-2.38, p<0.02, d=0.74$ $(0.19-1.34)]$.

\section{Network correlates of ReHo changes}

Clustered cell plots of correlations in functional connectivity (Fisher $z$-scores) between all 41 ReHo-ROIs in High and Low CRP-Glu groups are demonstrated in Fig. 4a, b. Significant differences were noted in correlation- and covariance-based comparisons of Fisher- $z$ connectivity matrix between High and Low CRP-Glu groups [Jennrich's Chi Sq(820) $=2235.65$ and Box Chi Sq $(861)=2645.61$, respectively, both $p<0.001]$. A MANOVA model with post hoc Benjamini-Hochberg correction $^{61}$ identified significant but modest reductions in "global efficiency" (reflecting greater network dysconnectivity) of a putative network combining all 41 ROIs measured in the High vs. Low CRP-Glu group [mean $(95 \% \mathrm{CI})=0.22(0.20-0.24)$ vs. $0.25(0.23-0.27), t(34)$ $=-2.10, p<0.04, d=0.66(0.03-1.280)]$.

\section{Disruptions in Subnetwork4 architecture associated with ReHo changes}

Multivariate ANCOVA followed by post hoc BenjaminiHochberg correction indicated that Subnetwork4 mean ReHo positively predicted global efficiency and node strength $(p$-corr $=0.009$ and 0.02 , respectively) and 
negatively predicted network diameter, path length and radius ( $p$-corr $=0.01,0.01$, and 0.03 , respectively) in the groups combined (Supplementary Table 4). Subnetwork4 assortativity (indicating decreasing network resilience) was also associated with psychomotor slowing indexed by prolongation of $5 \mathrm{CMT}[\mathrm{PE}=0.47, t=3.46$, FDR $p=$ $0.004, d=0.77(0.09-1.40)]$. Network eccentricity measures, i.e., diameter and radius (reflecting greater nodal segregation) positively predicted severity of scores on IDS-SR item\#30 "paralysis" [PE $=1.01, t=3.55, \mathrm{FDR} p<$ $0.001, d=1.18(0.60-1.84)$ and PE $-1.02, t=-3.6$, FDR $p$ $<0.001, d=1.24(0.55-1.88)$ for diameter and radius, respectively].

\section{Exploratory path analysis linking CRP-Glu, ReHo, network metrics, and behavior}

A path analysis based on Structural Equation Modeling was used to examine links among ReHo, network metrics and anhedonia. The pathway from Subnetwork 4 ReHo $\rightarrow$ Subnetwork4 measure $\rightarrow$ anhedonia was examined independently in the combined (both), and the High and Low CRP-Glu groups separately (Fig. 4c, d). A significant effect for the path extending from Subnetwork 4 ReHo $\rightarrow$ network eccentricity (diameter, radius) $\rightarrow$ anhedonia was seen only in the Low [diameter path coef $(95 \% \mathrm{CI})=0.14$ $(0.33-0.24), \quad z=2.58, \quad p=0.01, \quad$ and radius $=[0.13$ (0.006-0.26, $z=2.06, p=0.04)]$ but not among High or combined CRP-Glu groups (both $p>0.05$ ). SEM models replacing diameter with radius yielded identical results, while other measures associated with Subnetwork4 ReHo (global efficiency, network strength, path length) did not demonstrate statistically meaningful mediation effects.

\section{Discussion}

A distinct subtype of depressive behaviors including anhedonia and psychomotor slowing was revealed by CRP-Glu status. Behavior is an emergent property of coherent network activity patterns, and symptoms represent a failure of this function. It is unclear, if the lower limit of a critical minimum threshold of ordered, coherent local activity necessary to maintain network homeostasis is breached in the High (but not Low) CRPGlu group. As noted earlier, a disrupted balance between intra- vs. extrasynaptic signaling is likely to underlie the reduction in local homogeneity in the High CRP-Glu group ${ }^{18,24,62}$.

Stressed astroglial cells functioning under the duress of immune stimulation might lie at the epicenter of this network imbalance. Astroglial cells exist in assemblies that act as hubs that connect closely located but functionally divergent circuits (e.g., reward vs. aversion) by cross-synaptic dissemination of tonic, coherent, "near threshold" stimulation via their gliotransmission capabilities $^{63}$. The association between this tonic, coherent activity and local homogeneity is unclear but worth exploring. MRS primarily measures intracellular (astroglial) glutamate pool and hence increases in these signals might reflect increased cycling of glutamate or excessive intracellular pooling due to progressively declining astrocytic function $^{64,65}$. Of note, our previous MRS data in depressed patients indicate increased basal ganglia myoinositol (a putative marker of astroglial function) in association with increased CRP, possibly indicating a relationship between inflammation and astroglial dysfunction ${ }^{12}$.

Strengths of this study include use of multi-modal imaging techniques to compare key local and longdistance metrics of functional brain activity in a wellcharacterized, unmedicated group of subjects. Nevertheless, several limitations of the study need to be considered. Based on the aims and hypotheses, attention was focused on MRS glutamate signals from the left basal ganglia region-excluding other ROIs and other metabolites. However, a group comparison of the various other MRS metabolites did not reveal any group differences (Supplementary Table 2). Use of methods that estimate glutamine, lactate and glutamine-glutamate cycling such as ${ }^{13}$ carbon, hyperpolarized or ultra-high field MRS might have enabled a better appreciation of the underlying bioenergetic changes and helped discriminate glutamate dysregulation induced by astrocytic vs. neuronal pathologies. In addition, MRS provides limited spatial resolution that does not enable precise information about synaptic location or activity. However, MRS glutamate signals have consistently predicted neural activity in task- and transcranial magnetic stimulation (TMS)-activation based functional magnetic resonance imaging (fMRI) paradigms as well as functional connectivity in the resting-state ${ }^{66-68}$. Questions on mathematical modeling used to obtain $\mathrm{ReHo}$ (especially its non-linear origins) have been raised and clarified ${ }^{30,32,50,69}$. The adjusted power after control of covariates for the primary effects was robust (CRP-Glu prediction of MRS VOI $\mathrm{ReHo}=0.81$ and Subnetwork4 ReHo prediction of anhedonia $=0.94$ ). Nevertheless, the sample size was relatively small, and inferences drawn from the study could also be limited by its cross-sectional design. We have provided effect size estimates with uncertainty $(95 \% \mathrm{CI})$, applied bias-correction, corrected for multiple comparisons and wherever possible. Plasma CRP rather than other cytokines or CSF inflammatory markers was chosen for this study due to its overall stability, sensitivity and practical utility. Furthermore, as noted in another report from our group; plasma CRP demonstrated robust correlations with several other plasma inflammatory markers as well as CSF CRP justifying the focus on peripheral CRP ${ }^{70}$.

In summary, the findings point to disruption of local and long-distance functional brain activity in a subgroup 
of patients with both high inflammation and basal ganglia glutamate. The study illustrates the possibility of combining blood and neuroimaging-based biomarkers relevant to the pathophysiology of depression to identify biologically homogenous subtypes for research and personalized treatments. If replicated, ReHo might also emerge as a measure of target-engagement in the brain to test efficacy of glial stabilizers (riluzole), glutamate modulators (memantine, lamotrigine) or anti-inflammatory agents (infliximab), especially in conditions where connectivity and network metrics are extensively compromised.

\section{Acknowledgements}

This study was supported by grants R01MH H107033 \& K23MH091254 (Dr. Haroon), R01MH112076 (Drs. Haroon \& Miller), R01MH087604, R25MH101079 (Dr. Miller), and R01MH109637 (Dr. Felger) from the National Institute of Mental Health. In addition, the study was supported in part by PHS Grants UL1TR000454 and KL2TR000455 from the Clinical and Translational Science Award program, and by the $\mathrm{NIH/NCl}$ under award number P30CA138292. The funding sources had no role in the design and conduct of the study; collection, management, analysis, and interpretation of the data; preparation, review, or approval of the manuscript; and decision to submit the manuscript for publication. This work was supported in part by funding from a Shared Instrumentation Grant (S10) grant 1S100D016413-01 to the Emory University Center for Systems Imaging Core. We would like to acknowledge the help rendered by the staff and management of Emory University Center for Systems Imaging Core.

\section{Author details}

'Emory Behavioral Immunology Program, Winship Cancer Center, Emory University, Atlanta, GA 30322, USA. ²Department of Psychiatry \& Behavioral Sciences, Emory University School of Medicine, Atlanta, GA 30322, USA.

${ }^{3}$ Wallace H. Coulter Department of Biomedical Engineering, Emory University \& Georgia Institute of Technology, Atlanta, GA 30322, USA. ${ }^{4}$ School of Psychology and Sociology and Shenzhen Key Laboratory of Affective and Social Cognitive Science, Shenzhen University, Shenzhen 518060 Guangdong, People's Republic of China. ${ }^{5}$ Shenzhen Key Laboratory of Affective and Social Cognitive Science, Shenzhen University, Shenzhen 518060 Guangdong, People's Republic of China. ${ }^{6}$ Department of Anesthesiology, Emory University School of Medicine, Atlanta, GA 30322, USA. ${ }^{7}$ Department of Bioengineering, University of California, Riverside, CA 92521, USA

\section{Conflict of interest}

The authors declare that they have no conflict of interest.

\section{Publisher's note}

Springer Nature remains neutral with regard to jurisdictional claims in published maps and institutional affiliations.

Supplementary Information accompanies this paper at (https://doi.org/ 10.1038/s41398-018-0241-4).

Received: 31 July 2018 Revised: 31 July 2018 Accepted: 3 August 2018 Published online: 10 September 2018

\section{References}

1. Haroon, E., Raison, C. L. \& Miller, A. H. Psychoneuroimmunology meets neuropsychopharmacology: translational implications of the impact of inflammation on behavior. Neuropsychopharmacology 37, 137-162 (2012).

2. Wohleb, E. S., Franklin, T., Iwata, M. \& Duman, R. S. Integrating neuroimmune systems in the neurobiology of depression. Nat. Rev. Neurosci. 17, 497-511 (2016).
3. Wong, M. L. et al. Inflammasome signaling affects anxiety- and depressive-like behavior and gut microbiome composition. Mol. Psychiatry 21, 797-805 (2016).

4. Miller, A. H., Haroon, E. \& Felger, J. C. Therapeutic implications of brain-immune interactions: treatment in translation. Neuropsychopharmacology 42, 334-359 (2017).

5. Eisenberger, N. I. et al. Inflammation-induced anhedonia: endotoxin reduces ventral striatum responses to reward. Biol. Psychiatry 68, 748-754 (2010).

6. Harrison, N. A. et al. Inflammation causes mood changes through alterations in subgenual cingulate activity and mesolimbic connectivity. Biol. Psychiatry 66, 407-414 (2009).

7. Remus J. L., Dantzer R. Inflammation models of depression in rodents: Relevance to psychotropic drug discovery. Int. J. Neuropsychopharmacol. 19, (2016)

8. Miller, A. H., Haroon, E., Raison, C. L. \& Felger, J. C. Cytokine targets in the brain: impact on neurotransmitters and neurocircuits. Depress Anxiety 30, 297-306 (2013).

9. Savitz, J. et al. Activation of the kynurenine pathway is associated with striatal volume in major depressive disorder. Psychoneuroendocrinology 62, 54-58 (2015).

10. Menard, C. et al. Social stress induces neurovascular pathology promoting depression. Nat. Neurosci. 20, 1752-1760 (2017).

11. Haroon, E., Miller, A. H. \& Sanacora, G. Inflammation, glutamate, and glia: A trio of trouble in mood disorders. Neuropsychopharmacology 42, 193-215 (2017).

12. Haroon, E. et al. Conceptual convergence: increased inflammation is associated with increased basal ganglia glutamate in patients with major depression. Mol. Psychiatry 21, 1351-1357 (2016).

13. Haroon, E. et al. IFN-alpha-induced cortical and subcortical glutamate changes assessed by magnetic resonance spectroscopy. Neuropsychopharmacology 39, 1777-1785 (2014).

14. Haroon, E. et al. Age-related increases in basal ganglia glutamate are associated with TNF, reduced motivation and decreased psychomotor speed during IFN-alpha treatment: Preliminary findings. Brain Behav. Immun. 46 17-22 (2015)

15. Haroon, E. \& Miller, A. H. Inflammation effects on brain glutamate in depression: Mechanistic considerations and treatment implications. Curr. Top. Behav. Neurosci. 31, 173-198 (2017).

16. Dantzer, R. \& Walker, A. K. Is there a role for glutamate-mediated excitotoxicity in inflammation-induced depression? J. Neural Transm. Nienna) 121, 925-932 (2014).

17. Lewerenz, J. et al. The cystine/glutamate antiporter system $x(c)(-)$ in health and disease: from molecular mechanisms to novel therapeutic opportunities. Antioxid. Redox Signal. 18, 522-555 (2013).

18. Hardingham, G. E. \& Bading, H. Synaptic versus extrasynaptic NMDA receptor signalling: implications for neurodegenerative disorders. Nat. Rev. Neurosci. 11, 682-696 (2010).

19. Dantzer, R., O'Connor, J. C., Freund, G. G., Johnson, R. W. \& Kelley, K. W. From inflammation to sickness and depression: when the immune system subjugates the brain. Nat. Rev. Neurosci. 9, 46-56 (2008).

20. Schwarcz, R., Bruno, J. P., Muchowski, P. J. \& Wu, H. Q. Kynurenines in the mammalian brain: when physiology meets pathology. Nat. Rev. Neurosci. 13 465-477 (2012).

21. Piani, D. \& Fontana, A. Involvement of the cystine transport system xc- in the macrophage-induced glutamate-dependent cytotoxicity to neurons. J. Immunol. 152, 3578-3585 (1994).

22. Gras, G. et al. EAAT expression by macrophages and microglia: still more questions than answers. Amino acids 42, 221-229 (2012).

23. Rothstein, J. D. Excitotoxicity hypothesis. Neurology 47, S19-25 (1996).

24. Dorsett, C. R. et al. Glutamate neurotransmission in rodent models of traumatic brain injury. J. Neurotrauma 34, 263-272 (2016).

25. McCullumsmith, R. E. \& Sanacora, G. Regulation of extrasynaptic glutamate levels as a pathophysiological mechanism in disorders of motivation and addiction. Neuropsychopharmacology 40, 254-255 (2015).

26. Okubo, Y. \& lino, M. Visualization of glutamate as a volume transmitter. J. Physiol. 589(Pt 3), 481-488 (2011).

27. Biswal, B. B. Van Kylen, J. \& Hyde, J. S. Simultaneous assessment of flow and BOLD signals in resting-state functional connectivity maps. NMR Biomed. 10, 165-170 (1997)

28. Kannurpatti, S. S. \& Biswal, B. B. Detection and scaling of task-induced fMRIBOLD response using resting state fluctuations. Neuroimage 40, 1567-1574 (2008). 
29. Li, Z. et al. Alterations of resting-state $\mathrm{fMRI}$ measurements in individuals with cervical dystonia. Hum. Brain. Mapp. 38, 4098-4108 (2017).

30. Jiang, L. \& Zuo, X. N. Regional homogeneity: A multimodal, multiscale neuroimaging marker of the human connectome. Neuroscientist 22, 486-505 (2016).

31. Liu, Y. et al. Regional homogeneity associated with overgeneral autobiographical memory of first-episode treatment-naive patients with major depressive disorder in the orbitofrontal cortex: A resting-state fMRI study. J. Affect Disord. 209, 163-168 (2017).

32. Zang, Y., Jiang, T., Lu, Y., He, Y. \& Tian, L. Regional homogeneity approach to fMRI data analysis. Neuroimage 22, 394-400 (2004).

33. APA. Diagnostic and Statistical Manual of Mental Disorders, IV edn. (American Psychiatric Press, Washington, DC, USA., 2000).

34. First, M. B., Spitzer, R. L., Gibbon, M. \& Williams, J. B. Structured Clinical Interview for DSM-IV. (American Psychiatric Press, Washington DC, 1997).

35. Whitton, A. E., Treadway, M. T. \& Pizzagalli, D. A. Reward processing dysfunction in major depression, bipolar disorder and schizophrenia. Curr. Opin. Psychiatry 28, 7-12 (2015).

36. Rush, A. J. et al. The 16-ltem quick inventory of depressive symptomatology (QIDS), clinician rating (QIDS-C), and self-report (QIDS-SR): a psychometric evaluation in patients with chronic major depression. Biol. Psychiatry $\mathbf{5 4}$ 573-583 (2003)

37. Trivedi, M. H. et al. The inventory of depressive symptomatology, clinician rating (IDS-C) and self-report (IDS-SR), and the quick inventory of depressive symptomatology, clinician rating (QIDS-C) and self-report (QIDS-SR) in public sector patients with mood disorders: a psychometric evaluation. Psychol. Med. 34, 73-82 (2004).

38. Ameli, R. et al. SHAPS-C: the Snaith-Hamilton pleasure scale modified for clinician administration. PeerJ 2, e429 (2014).

39. Felger, J. C. et al. Inflammation is associated with decreased functional connectivity within corticostriatal reward circuitry in depression. Mol. Psychiatry 21, 1358-1365 (2016).

40. Collyer, C. E., Broadbent, H. A. \& Church, R. M. Preferred rates of repetitive tapping and categorical time production. Percept. Psychophys. 55, 443-453 (1994).

41. Reitan, R. M. The relation of the trail making test to organic brain damage. J. Consult. Psychol. 19, 393-394 (1955).

42. Wechsler, D. Wechsler Adult Intelligence Test. 4th edn, (Pearson, San Antonio, TX, USA, 2008).

43. Robbins, T. W. et al. A study of performance on tests from the CANTAB battery sensitive to frontal lobe dysfunction in a large sample of normal volunteers: implications for theories of executive functioning and cognitive aging. J. Int Neuropsychol. Soc. 4, 474-490 (1998).

44. Sahakian, B. J. \& Owen, A. M. Computerized assessment in neuropsychiatry using CANTAB: discussion paper. J. R. Soc. Med. 85, 399-402 (1992).

45. Heberlein, K. A. \& Hu, X. Simultaneous acquisition of gradient-echo and asymmetric spin-echo for single-shot z-shim: Z-SAGA. Magn. Reson. Med. 51, 212-216 (2004).

46. Provencher SK. LCModel and LCMgui User's Manual. pp. 1-174 (LCMODEL). Steven K. Provencher (2016).

47. Cox, R. W. AFNl: Software for analysis and visualization of functional magnetic resonance neuroimages. Comput. Biomed. Res. 29, 162-173 (1996). Software available at. https://afni.nimh.nih.gov.

48. Alexopoulos, G. \& Morimoto, S. The inflammation hypothesis in geriatric depression. Int. J. Geriatr. Psychiatry 26, 1109-1118 (2011)
49. Benedetti, F. et al. Inflammatory cytokines influence measures of white matter integrity in Bipolar Disorder. J. Affect Disord. 202, 1-9 (2016).

50. Zang, Y. F. et al. Altered baseline brain activity in children with ADHD revealed by resting-state functional MRI. Brain. Dev. 29, 83-91 (2007).

51. Zou, Q. H. et al. An improved approach to detection of amplitude of lowfrequency fluctuation (ALFF) for resting-state fMRI: fractional ALFF. J. Neurosci. Methods 172, 137-141 (2008).

52. Eklund, A., Nichols, T. E. \& Knutsson, H. Cluster failure: Why fMRI inferences for spatial extent have inflated false-positive rates. Proc. Natl Acad. Sci. USA 113, 7900-7905 (2016).

53. Cox, R. W., Chen, G., Glen, D. R., Reynolds, R. C. \& Taylor, P. A. FMRI clustering in AFNl: False-positive rates redux. Brain Connect. 7, 152-171 (2017).

54. Rubinov, M. \& Sporns, O. Complex network measures of brain connectivity: uses and interpretations. Neuroimage 52, 1059-1069 (2010).

55. Sporns, O. Networks of the Brain. (The MIT Press, Cambridge, MA, 2011).

56. Drysdale, A. T. et al. Resting-state connectivity biomarkers define neurophysiological subtypes of depression. Nat. Med. 23, 28-38 (2017).

57. Cohen, J. Statistical Power Analysis for the Behavioral Sciences. 2nd edn (L. Erlbaum Associates, Hillsdale, N.J., 1988). xxi, 567 p.

58. Power, J. D. et al. Functional network organization of the human brain. Neuron 72, 665-678 (2011).

59. Kaiser, R. H., Andrews-Hanna, J. R., Wager, T. D. \& Pizzagalli, D. A. Large-scale network dysfunction in major depressive disorder: A meta-analysis of restingstate functional connectivity. JAMA Psychiatry 72, 603-611 (2015).

60. Haber, S. N. \& Knutson, B. The reward circuit: linking primate anatomy and human imaging. Neuropsychopharmacology 35, 4-26 (2010).

61. Benjamini, Y., Hochberg, Y. Controlling the false discovery rate: a practical and powerful approach to multiple testing. J. R. Stat. Soc. Ser. B (Methodol.). 57, 289-300 (1995)

62. Shan, D., Yates, S., Roberts, R. C. \& McCullumsmith, R. E. Update on the neurobiology of schizophrenia: a role for extracellular microdomains. Minerva Psichiatr. 53, 233-249 (2012).

63. Rossi, D. Astrocyte physiopathology: At the crossroads of intercellular networking, inflammation and cell death. Prog. Neurobiol. 130, 86-120 (2015).

64. Mason, G. F. \& Krystal, J. H. MR spectroscopy: its potential role for drug development for the treatment of psychiatric diseases. NMR Biomed. 19, 690-701 (2006).

65. Rothman, D. L., De Feyter, H. M., de Graaf, R. A., Mason, G. F. \& Behar, K. L. $13 C$ MRS studies of neuroenergetics and neurotransmitter cycling in humans. NMR Biomed. 24, 943-957 (2011).

66. Horn, D.I. et al. Glutamatergic and resting-state functional connectivity correlates of severity in major depression - the role of pregenual anterior cingulate cortex and anterior insula. Front Syst Neurosci. 4, 1-10 (2010).

67. Rowland, L. M. et al. Frontal glutamate and gamma-aminobutyric acid levels and their associations with mismatch negativity and digit sequencing task performance in schizophrenia. JAMA Psychiatry 73, 166-174 (2016).

68. Walter, M. et al. The relationship between aberrant neuronal activation in the pregenual anterior cingulate, altered glutamatergic metabolism, and anhedonia in major depression. Arch. Gen. Psychiatry 66, 478-486 (2009).

69. Zhang, B. et al. Altered functional connectivity density in major depressive disorder at rest. Eur. Arch. Psychiatry Clin. Neurosci. 266, 239-248 (2016).

70. Felger, JC. et al. What does plasma CRP tell us about peripheral and centra inflammation in depression? Mol Psychiatry. https://doi.org/10.1038/s41380018-0096-3 (2018). 